\title{
Fiber Segment Interferometry for Dynamic Strain Measurements
}

\author{
Thomas Kissinger, Ricardo Correia, Thomas O. H. Charrett, Stephen W. James, and Ralph P. Tatam
}

\begin{abstract}
Using a novel range-resolved interferometric signal processing technique based on the sinusoidal optical frequency modulation of a cost-effective laser diode, a fiber sensing approach termed fiber segment interferometry (FSI) is described. In FSI, a chain of long-gauge length fiber optic strain sensors are separated by identical in-fiber partial reflectors. Targeted at dynamic strain analysis and ultrasound detection for structural health monitoring, this approach allows integrated strain measurements along fiber segments, removing the sensing gaps and sensitivity to inhomogeneities found with localized fiber sensors. In this paper, the multiplexing of six fiber segments, each of length $12.5 \mathrm{~cm}$, is demonstrated. The sensor array can be interrogated at $98 \mathrm{kHz}$ data rate, achieving dynamic strain noise levels $\leq 0.14 \mathrm{n} \epsilon \cdot \mathrm{Hz}^{-0.5}$. The reflector fabrication is discussed, an analysis of linearity and noise performance is carried out and results from an exemplar experiment to determine the speed-of-sound of a stainless steel rod are shown.
\end{abstract}

Index Terms - Condition monitoring, fiber gratings, optical fiber sensors, optical interferometry, strain measurement.

\section{INTRODUCTION}

$\mathbf{L}$ ONG-GAUGE length interferometric fiber optic strain sensors [1] integrate the applied strain field over the sensing fiber, which contrasts with widely-used localised fiber optical strain sensors, such as fiber Bragg gratings (FBGs) [2]. The advantages of long-gauge length sensors for structural health monitoring include the reduced dependence on the local properties at the sensor location of measurements made on inhomogeneous substrates [3] and the absence of sensing gaps, whereby the integrating property of the measurement ensures that a localised strain event anywhere along the sensing fiber will not be missed. Long-gauge length fiber optical strain sensors have been researched intensively for use in hydrophones [4]-[7], where high-specification, high cost lasers are used to facilitate low noise operation and where sensor gauge lengths are typically tens of meters. In contrast, for applications in structural health monitoring that are targeted by this study, sensor gauge lengths

Manuscript received December 15, 2015; revised February 12, 2016; accepted February 12, 2016. Date of publication February 17, 2016; date of current version September 22, 2016. This work was supported by the Engineering and Physical Sciences Research Council U.K., under grants EP/H02252X/1 and EP/G033900/1.

T. Kissinger, T. O. H. Charrett, S. W. James, and R. P. Tatam are with Engineering Photonics, Cranfield University, Bedford MK43 0AL, U.K. (e-mail: t.kissinger@cranfield.ac.uk; t.charrett@cranfield.ac.uk; s.w.james@cranfield. ac.uk; r.p.tatam@cranfield.ac.uk).

R. Correia was with Cranfield University, Bedford MK43 0AL, U.K. He is now with Electrical and Electronic Engineering, University of Nottingham, Nottingham NG7 2RD, U.K. (e-mail: ricardo.goncalvescorreia@nottingham. ac.uk).

Color versions of one or more of the figures in this paper are available online at http://ieeexplore.ieee.org.

Digital Object Identifier 10.1109/JLT.2016.2530940

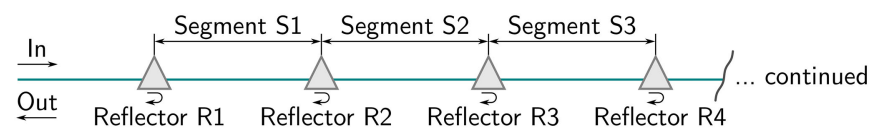

Fig. 1. Illustration of the FSI approach, where equal-length fiber segments spanned between identical in-fiber partial reflectors form an array of long-gauge length fiber optic strain sensors.

in the tens of metres range would be considered impractical for many potential applications, while the cost-effectiveness and the practicality of the construction and use of the sensing fiber may be more important factors. Here the proposed approach offers considerable advantages, such as sensor gauge lengths in the $10 \mathrm{~s}$ of $\mathrm{cm}$ range. As currently constructed, the interferometric approach proposed in this paper can only measure relative strain changes from an a priori unknown starting point, and is thus only suited for purely dynamic strain sensing applications, while further developments could also allow absolute strain/temperature sensing in the future. Dynamic strain measurement applications feasible with the current implementation include vibration-based condition monitoring [8], [9], cyclic load estimation [10], rotor imbalance detection [11] and the monitoring of transient strain events, such as quench detection in superconducting magnets [12] or impact detection [13], [14]. Also applications in fiber-optic ultrasound detection using integrating long-gauge length sensors [15], [16] could be envisaged.

In this work, we apply a recently developed range-resolved interferometric signal processing technique [17] to long-gauge length strain sensing in an approach termed fiber segment interferometry (FSI). In FSI, a chain of fiber segments that act individually as interferometric, long-gauge length fiber optic sensors are formed by pairs of identical, in-fiber partial reflectors, as illustrated in Fig. 1. Previously, we have presented an FSI technique based on the use of code-division multiplexing [18] to interrogate fiber segments of length $16.5 \mathrm{~m}$. Using the new signal processing technique [17], this can now be reduced by over two orders of magnitude to $12.5 \mathrm{~cm}$. In this new approach, sinusoidal modulation of the injection current of a cost-effective DFB laser diode of the type used in telecommunications is employed to produce the necessary optical frequency modulation, removing the need for an external modulator. Also, the use of optical frequency modulation allows the interrogation of self-referencing interferometric configurations that provide high stability.

Apart from FSI implementations for hydrophone applications [4]-[6], recent FSI applications in distributed sensing include the work by Huang et al. [19]. In [19], optical carrier based microwave interferometry was used to allow the multiplexing of fiber segments of gauge lengths of $12 \mathrm{~cm}$, similar to the segment 
length in this paper. However, an external modulator and signal processing hardware capable of $6 \mathrm{GHz}$ input bandwidth had to be used, which is two orders of magnitude larger than the $50 \mathrm{MHz}$ input bandwidth used in this paper. This also illustrates a key advantage of the proposed range-resolved signal processing technique, namely the decoupling of the spatial resolution (gauge length) from the signal processing hardware bandwidth [17]. A concept related to FSI was proposed by Wang et al. [20], where nested Fabry-Perot sensors are created between weak, broadband FBG-based partial reflectors, similar to those reflectors used in this work. Recently, Chen et al. [21] reported the interrogation of four fiber segments that formed an FSI-type array using femtosecond inscribed reflectors. However, both of these approaches [20], [21], while achieving much higher spatial resolutions in the millimetre and sub-millimetre ranges, use widely tunable lasers and therefore lack the simplicity and cost-effectiveness of the work presented in this paper. FSI-based sensing also competes with high-density quasi-distributed FBG sensing, where large numbers of identical FGBs are interrogated [22], [23], or fully distributed techniques based on the evaluation of Rayleigh [24], [25] or Brillouin scattering [26]. A key advantage of FSI over quasi-distributed FBG sensing is the simplicity of using a monolithic laser diode. Compared to techniques involving the measurement of backscatter from the fiber, FSI allows quantitative measurements of strain within a precisely defined measurement region.

In this paper, expanding work first reported at the 24th Optical Fiber Sensors Conference, OFS24 [27], we present a complete description of the system that was used to interrogate six FSI segments of gauge length $12.5 \mathrm{~cm}$ at a data rate of $98 \mathrm{kHz}$. The properties of the low-reflectivity, broadband FBGs that were used in this work as in-fiber partial reflectors are discussed and the noise and linearity performance is analysed. The capabilities of the system are demonstrated by a measurement of the transient propagation delays of an acoustic pulse traversing a stainless steel rod, allowing the determination of the speed-of-sound in the rod, before possible future improvements to the approach are discussed in detail.

\section{SETUP AND IMPLEMENTATION}

\section{A. Signal Processing and Optical Setup}

The range-resolved signal processing approach [17] employed in this work uses a sinusoidal optical frequency modulation waveform of modulation frequency $f_{\bmod }$ and optical frequency excursion amplitude $f_{\text {opt }}$. For an interferometer with non-zero optical path difference (OPD), this results in a sinusoidal phase modulation waveform of phase carrier amplitude $A$, with $A$ being approximately proportional to the OPD of the interferometer and to $f_{\mathrm{opt}}$. The phase modulation produces a characteristic photo detector signal for each interferometer present in the system. For several constituent interferometers, these signals superimpose linearly, allowing the interferometer signals to be separated according to their OPD. The key steps in this signal processing approach, described fully in [17], are the multiplicative application of a smooth window function to the photo detector signal and the demodulation with

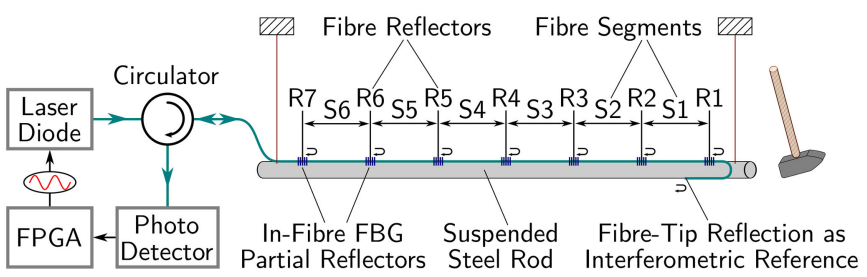

Fig. 2. Experimental setup used to measure acoustic wave propagation after a hammer strike. The FPGA processor sinusoidally modulates the injection current of the laser diode. This light is guided using a circulator to an optical fiber mounted on a freely suspended steel rod, where seven partial reflectors $\mathrm{R} 1$ to $\mathrm{R} 7$ form six fiber segments $\mathrm{S} 1$ to $\mathrm{S} 6$ of gauge length $12.5 \mathrm{~cm}$. The light returned by the reflectors, along with the interferometric reference provided by the fiber tip, reaches the photo detector and the resulting interference signals are demodulated by the FPGA.

a time-variant carrier that, for each range channel, resembles the complex version of the photo detector signal expected for that range. Together, both of these measures result in a rangeresolved interferometric signal processing technique that allows very linear interferometric phase evaluation. Unlike prior work [5], where the OPDs of constituent interferometers were required to adhere to integer OPD ratios, this approach allows completely variable OPD placement of signal sources, subject to a minimum separation, greatly increasing its flexibility and practicality. Furthermore, the non-idealities resulting from the use of laser injection current modulation, such as associated intensity modulation and non-linear optical frequency modulation, can be corrected straightforwardly [17].

The experimental setup used is illustrated in Fig. 2. Here the FSI sensing fiber, consisting of six fiber segments (S1 to S6), each of physical length $12.5 \mathrm{~cm}$, formed between seven in-fiber FBG partial reflectors (R1 to R7), is epoxy bonded to a freely suspended stainless steel rod (type 316 stainless steel, length: $78.2 \mathrm{~cm}$, diameter: $1.9 \mathrm{~cm}$ ). The interferometric reference, or local oscillator (LO), is formed by the Fresnel reflection from the cleaved fiber tip, providing a simple, self-referencing configuration with complete down-lead insensitivity and constituting a low-finesse Fabry-Perot interferometric configuration. The optical setup comprises a $1550 \mathrm{~nm}$ laser diode (Eblana Photonics EP1550-NLW-B), an optical fiber circulator and an InGaAs photo detector. The field programmable gate array (FPGA)based real-time signal processing implementation is comparable to the one described previously [17], operating at a clock frequency of $100 \mathrm{MHz}$ for the digital data acquisition and signal processing. As in [17], the modulation frequency is $f_{\bmod }=$ $98 \mathrm{kHz}$ and the optical frequency excursion amplitude is $f_{\mathrm{opt}}=$ $9 \mathrm{GHz}(\approx 0.07 \mathrm{~nm}$ wavelength tuning amplitude). Fig. 3 plots the photo detector signal recorded over one modulation period and also shows the resulting signal after the intensity modulation associated with injection current modulation has been corrected as described in [17], using the same correction parameters. The demodulation window function, a dual Gaussian window function [17] of width parameter $\sigma=0.025$, is also shown in Fig. 3 .

After demodulation with the time-variant carrier [17], the range dependency of the complex quadrature signal amplitude can be plotted as a function of the demodulation phase carrier amplitude $A_{d}$, as shown in Fig. 4. Here, the signals originating 


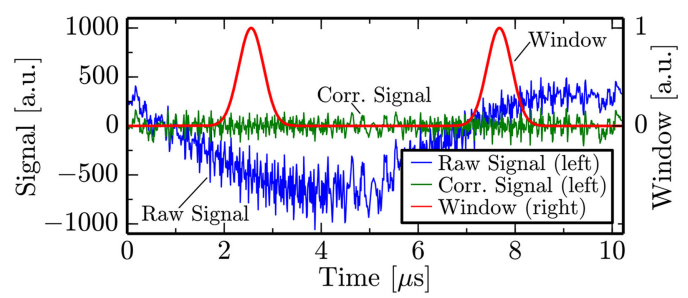

Fig. 3. Photo detector signals before and after correction of the undesired intensity modulation, while the secondary $y$-axis shows the dual Gaussian window function applied during the signal processing.

Corresponding Range $[\mathrm{cm}]$

$\begin{array}{lllllllll}0 & 18.8 & 31.3 & 43.8 & 56.3 & 68.8 & 81.3 & 93.8\end{array}$

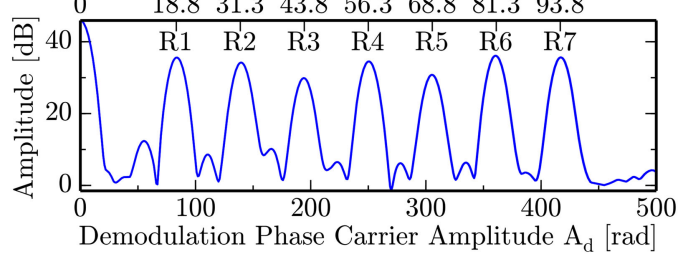

Fig. 4. Plot of the quadrature signal amplitude as a function of the demodulation phase carrier amplitude $A_{d}$, with the corresponding range shown on the secondary $x$-axis and the peaks from reflectors R1 to R7 labelled.

from the interferometers formed between the seven reflectors R1 to R7 and the fiber tip reference can be clearly identified. The secondary $x$-axis of Fig. 4 shows the corresponding physical distances of the reflectors from the fiber tip. Following the computation of the complex quadrature signals, low-pass filtered at a bandwidth of $43 \mathrm{kHz}$, for each of the reflectors R1 to R7, the interferometric phases can be extracted from the quadrature signals at a data rate equal to $f_{\bmod }$ at $98 \mathrm{kHz}$ and subjected to phase unwrapping [28]. Finally, the signals from adjacent reflectors that form a segment are subtracted to obtain the desired phase signals for segments S1 to S6.

In this work, a segment length, $l_{s}$, of $12.5 \mathrm{~cm}$ was chosen, resulting in a phase carrier amplitude difference of $56 \mathrm{rad}$ between the return signals from adjacent reflectors for the optical frequency excursion amplitude, $f_{\mathrm{opt}}$, of $9 \mathrm{GHz}$ used. This is approximately a factor of two more than the value of $A_{\mathrm{m} \text { in }}$ required according to the spatial resolution calculation for a specified baseband suppression value of $-50 \mathrm{~dB}$ at $\sigma=0.025$ [17]. Additionally, an offset distance of $1.5 l_{s} \approx 18.8 \mathrm{~cm}$ between the fiber tip and the first reflector R1 was introduced. The result of both these measures is that OPDs of undesired signals, corresponding to the mutual interference between reflectors and thus having phase carrier amplitudes that are direct multiples of $56 \mathrm{rad}$, are interleaved with the desired signals at $1.5 \cdot 56 \mathrm{rad}=84 \mathrm{rad}, 84 \mathrm{rad}+56 \mathrm{rad}=140 \mathrm{rad}$, etc. Therefore, crosstalk from undesired sources is spatially offset from the desired ranges and is effectively suppressed. The undesired signal peaks are also visible in Fig. 4 at $A_{d}=56 \mathrm{rad}, 112 \mathrm{rad}$, etc. This procedure comes at the price of an increase by a factor of two in the minimally permissible spatial separation between reflectors. A further option to increase suppression of parasitic signals due to mutual interference between reflectors would be to increase the reflectivity of the fiber tip using an appropriate coating.

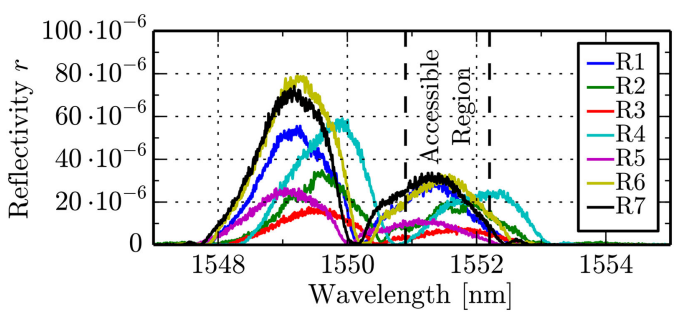

Fig. 5. The reflectivity spectra of the seven FBGs R1 to R7, measured by the Luna OBR 4400 Reflectometer, are shown. Here, a region of spectral width $1.3 \mathrm{~nm}$ that is accessible by temperature tuning of the laser diode is marked.

\section{B. In-Fiber Reflectors}

In general, in-fiber partial reflectors used in FSI need to be sufficiently broadband to return signals under all conceivable local strain and temperature conditions, meaning that FBG-based reflectors should have a spectral width lying in the range of 1 to $10 \mathrm{~nm}$. A $1 \mathrm{~nm}$ spectral width would allow measurements over a temperature range of $\approx 100 \mathrm{~K}$ or, alternatively, over a strain range of $\approx 0.1 \%$, while a $10 \mathrm{~nm}$ spectral width would allow operation over $\approx 1000 \mathrm{~K}$ temperature or $\approx 1 \%$ strain ranges [2]. In FSI, the use of in-fiber partial reflectors produced from mechanical splices [4], coupler-based reflectors [6] or FBGs [29] have been demonstrated. Additionally, reflective fusion splices [30] or microcavities [31] could also be considered. FBGs represent an attractive in-fiber partial reflector because they can be inscribed cost-effectively, do not require breakage of the fiber and are not bulky. While chirped FBG [32] would offer return spectra of appropriate width, the location of the point of reflection of a particular wavelength in a chirped FBGs moves with changes in strain or temperature. This would introduce an additional OPD change into the segment phase signals, making chirped gratings unsuitable for FSI. For non-chirped FBGs, the spectral width generally broadens with decreasing length. Non-chirped broadband FBGs of suitable quality for FSI, with a length of $16 \mu \mathrm{m}$, a full-width half maximum spectral width of $50 \mathrm{~nm}$ and a reflectivity of $r=10^{-4}$ have also been fabricated in hydrogenated standard SMF-28 fiber by Wang et al. [20].

The non-chirped FBGs used here were inscribed in nonhydrogenated SMF-28 fiber using a frequency-quadrupled Nd-YAG laser emitting at a wavelength of $266 \mathrm{~nm}$. A slit of width of $1.5 \mathrm{~mm}$ was placed $24 \mathrm{~cm}$ from a phase mask with the fiber located $2 \mathrm{~mm}$ behind the phase mask. During FBG inscription the evolution of the Bragg reflection was monitored in real time using the FSI signal processing implementation. The final FBG spectra for reflectors R1 to R7 were then measured using a Luna OBR 4400 Reflectometer and are shown in Fig. 5. These spectra reach peak reflectivities, $r$, of up to $0.008 \%$ and show two distinct regions of $\approx 2 \mathrm{~nm}$ spectral width each, separated by a region where $r$ drops to near zero. A region of $1.3 \mathrm{~nm}$ width, marked in Fig. 5, is accessible to the experiment through temperature tuning of the laser emission wavelength and it will be demonstrated later that successful measurements can be taken anywhere in that region. This is possible because, even though the reflectivities of the FBGs are low, they are still significantly above the Rayleigh scattering background, and the heterodyne gain from the interferometric fiber-tip reference effectively am- 


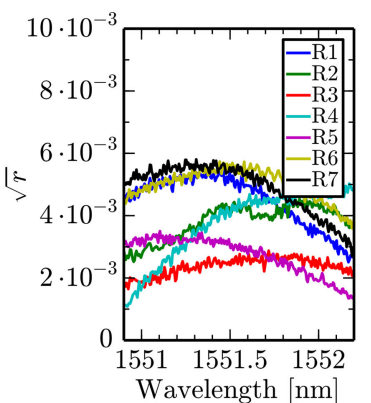

(a)

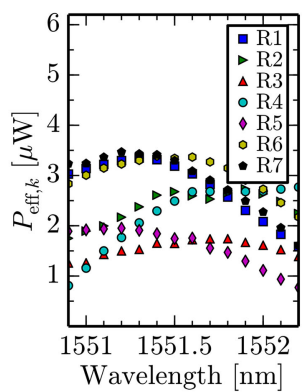

(b)
Fig. 6. The square root of the reflectivity spectra for FBGs R1 to R7, measured by the Luna OBR 4400 Reflectometer, over the accessible region marked in Fig. 5 is shown in (a), while (b) shows the effective signal powers for the FBGs as a function of wavelength measured by the proposed technique.

plifies the weak return signals. For future implementations, it appears entirely feasible to optimize FBG inscription to obtain the favourable FBG qualities described by Wang et al. [20]. However, employing non-optimised FBG reflectors with irregular spectra and very low reflectivity levels is very useful to demonstrate the robustness of the FSI approach. Therefore no further optimisation of the FBG properties was attempted in this study.

\section{EXPERIMENTAL RESULTS}

\section{A. FBG Return Signals}

As an interferometric technique, the amplitudes of the complex quadrature signals are proportional to the effective powers, $P_{\text {eff }, k}$, for each of the $k$ reflectors. Thus $P_{\text {eff }, k} \propto \sqrt{P_{\mathrm{LO}} P_{k}}$, where $P_{\mathrm{LO}}$ is the power returned from the LO, i.e. the fiber tip reflection in the setup of Fig. 2, and $P_{k}$ is the return power for the $k$ th reflector. $P_{\text {eff }, k}$ is also dependent on the mutual polarisation and coherence overlap between the light returned from the FBG in-fiber partial reflector and the LO. For a fixed LO power, $P_{\mathrm{LO}}$, and stable polarisation this means that the measured signals are proportional to the square root of the reflectivity $\sqrt{r}$ of each reflector. A plot of $\sqrt{r}$ measured by the Luna OBR 4400 Reflectometer over the accessible region, marked in Fig. 5(a), is shown in Fig. 6(a). This is for comparison with Fig. 6(b), which plots the effective powers, $P_{\text {eff }, k}$, measured directly by the proposed approach and where the laser emission centre wavelength was adjusted by temperature tuning. Here, the signal processing implementation-specific units of the quadrature signal amplitudes were calibrated into physical power units in $\mu \mathrm{W}$ using a dedicated free-space setup, where the powers in the interferometric arms could be measured individually and full polarisation and coherence overlap was maintained. The similarity between Fig. 6(a) and (b) illustrates that, for a stable LO power, the relevant signal powers are indeed dependent on the square root of the reflectivities of the partial in-fiber reflectors and also highlights that this approach could also be used for range-resolved interrogation of regular FBG or Fabry-Perot sensor return spectra.

\section{B. Linearity and Crosstalk}

The quadrature signals traces resulting from an acoustic excitation of the steel rod, captured over $20 \mathrm{~ms}$ for the seven reflec-

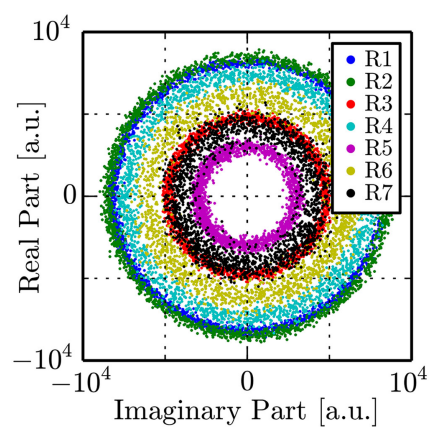

Fig. 7. The time traces of the quadrature signals for the seven reflectors R1 to R7 are shown in the complex plane, recorded over a period of $20 \mathrm{~ms}$ during acoustic excitation of the steel rod.

tors R1 to R7, are shown in Fig. 7 in the complex plane. Here, the quadrature amplitudes for the different reflectors vary by up to a factor of three and the resulting quadrature signals can be seen to be both circular and concentric, where both circularity and concentricity are requirements for interferometric demodulation without cyclic errors. In order to quantify the magnitude of the cyclic errors for each reflector, the quadrature amplitudes were averaged over 30 angular sectors. When the Heydemann cyclicerror ellipse [33] is fitted to this, maximum quadrature amplitude deviations from the mean quadrature amplitudes ranging between $0.1 \%$ for R5 and $0.7 \%$ for R7, with corresponding cyclic error amplitudes ranging between 1 and $7 \mathrm{mrad}$, were observed.

The characterisation of crosstalk between fiber segment sensors requires the ability to induce test signals in one fiber segment and observe any crosstalk in the phase signals of other segments while these are physically isolated from the test signal source. In this study, the FBG-based reflectors were bonded to the steel rod immediately after fabrication, therefore it was not possible to achieve physical isolation of the sensors and so no study of crosstalk was performed. However, it is known from previous experiments using this signal processing approach that digital quantisation errors in the signal processing can cause both cyclic errors and crosstalk in the quadrature signals that are of similar magnitudes [34]. Therefore, the quadrature signal crosstalk can be estimated to be of the order of -60 to $-40 \mathrm{~dB}$, equivalent to quadrature amplitude deviations on the order of $0.1 \%$ and $1 \%$, respectively, that were determined in Fig. 7. In general, it is important to note that crosstalk in the fiber segment phase signals, which is the relevant quantity in FSI, is non-trivially dependent on the quadrature signal amplitudes, bias phases as well as on the phase signal amplitudes and frequencies of the participating reflector signals. However, in typical practical situations with large amplitude phase signals ( $\gg 2 \pi \mathrm{rad}$ ) it is often found to be much lower numerically than the corresponding quadrature signal crosstalk [18], [34].

\section{Noise}

The unwrapped phase signals of both the reflector and the resulting segment signals exhibit a mostly flat noise floor over the entire $43 \mathrm{kHz}$ quadrature bandwidth, therefore allowing the quantification of noise levels in units of $\mathrm{rad} \cdot \mathrm{Hz}^{-0.5}$. For the experiments in this section, this is calculated by dividing the standard deviation of the phase signal, measured without 


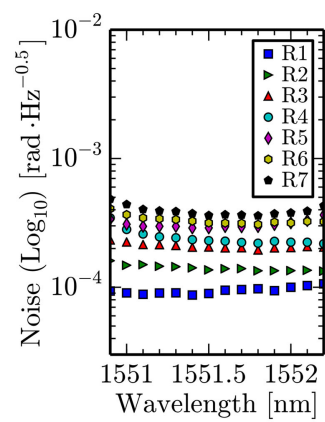

(a)

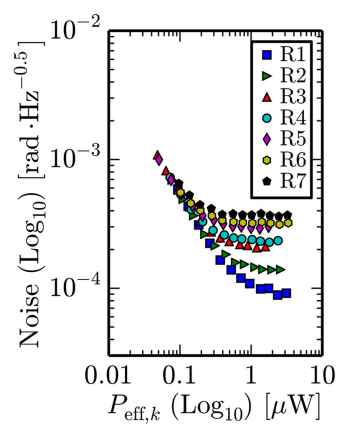

(b)
Fig. 8. For each reflector R1 to R7, (a) plots the dependency of the noise levels on the interrogation wavelength and (b) plots the dependency of the noise levels on the effective power.

any excitation of the rod over an acquisition time of $100 \mathrm{~ms}$, by the square root of the bandwidth. For each reflector, these noise levels are then quantified in Fig. 8(a) as a function of interrogation wavelength. Here it can be seen that the noise levels stay broadly constant over the entire accessible wavelength region. The remaining wavelength dependency roughly correlates with reductions in the effective powers of the reflections evident in Fig. 6(b). Therefore successful operation over the entire $1.3 \mathrm{~nm}$ wavelength region used in this study has been confirmed, even though the reflectivities, as evident in Fig. 5, vary significantly over this wavelength range, for example by up to a factor of 20 for reflector R4.

In Fig. 8(b), the return powers were artificially attenuated by inducing bend loss in the fiber lead connecting to the sensing array of Fig. 2, allowing the noise levels to be plotted as a function of effective power, $P_{\mathrm{eff}, k}$, for each reflector. In this experiment, the interrogation wavelength was fixed at $1551.5 \mathrm{~nm}$, where it can be seen in Fig. 6(b) that the effective powers of all reflectors are generally high. Fig. 8(a) shows that, while the power is reduced, the noise levels for each reflector initially stays at a constant plateau value until these plateaus asymptotically merge into a common, sloped line. Therefore, it can be concluded that the experiment in which the reflections are not artificially attenuated is dominated by a noise source that is independent of the return signal power, such as laser phase noise. Conversely, the decreasing effective powers associated with the artificial attention increase the relative contributions of other noise sources such as shot, intensity, electronic and detector noise, leading to the asymptotic behaviour observed in Fig. 6(b).

The dominance of laser phase noise in these measurements is further confirmed by Fig. 9(a), which plots the noise dependency of the reflector signals on the OPD, again at a fixed wavelength of $1551.5 \mathrm{~nm}$ and without any artificial attenuation. Here, a linear least-square fit of these values is drawn using a continuous line, with a slope value of $1.2 \cdot 10^{-4} \mathrm{rad} \cdot \mathrm{Hz}^{-0.5} \cdot \mathrm{m}^{-1}$, illustrating the strong dependence of the noise levels on the OPD. The small offset of $3.0 \cdot 10^{-5} \mathrm{rad} \cdot \mathrm{Hz}^{-0.5}$ of this fit at zero OPD could have several origins: it may be caused by the previously discussed onset of noise sources such as shot, intensity, electronic and detector noise or it could result from high-frequency laser phase noise that is aliased back into the signal band [35]

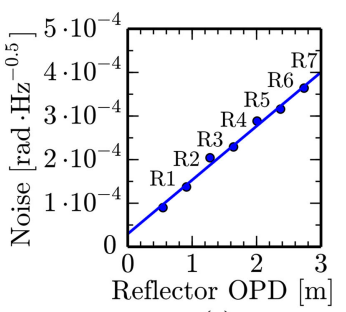

(a)

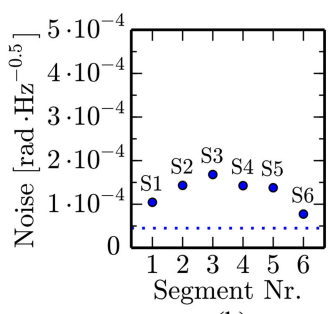

(b)
Fig. 9. (a) plots the OPD dependence of the noise levels of the reflector signals R1 to R7, with a linear fit drawn using a continuous line, while (b) plots the noise levels in the segment signals S1 to S6, with the theoretical phase noise level indicated using the dotted line.

and thus appears as a common noise background in the quadrature signals of all reflectors.

Finally, the noise levels in the segment signals S1 to S6 are plotted in Fig. 9(b), again computed over the entire $43 \mathrm{kHz}$ quadrature bandwidth. Theoretically, for the case of complete laser phase noise domination and ideal signal processing, the expected segment noise levels should be identical for each segment and depend only on the segment OPD. The segment OPD is given by $2 l_{s} n_{g}$, with group index of refraction $n_{g}=1.46$, which, using the OPD slope value determined previously, results in an expected segment noise level of $4.5 \cdot 10^{-5} \mathrm{rad} \cdot \mathrm{Hz}^{-0.5}$, with this value marked using the dotted line in Fig. 9(b). However, it can be seen that the measured segment noise levels exceed this theoretical limit, by up to a factor of four for segment S3, and similar behaviour is found for all interrogation wavelengths over the $1.3 \mathrm{~nm}$ accessible region. The potential reasons for this excess noise in the segment signals are manifold and could be due to fundamental noise sources, such as the previously mentioned aliased high-frequency laser phase noise [35], or, alternatively, from the measurement of genuine broadband environmental acoustic noise. As physical origins cannot be ruled out, no definitive answer can be given at this stage and a further detailed investigation is required. Regardless of this, it can be concluded that interrogation with segment noise levels $1.7 \cdot 10^{-4} \mathrm{rad} \cdot \mathrm{Hz}^{-0.5}$ has been demonstrated, which, for a $12.5 \mathrm{~cm}$ segment corresponds [18] to strain noise levels of $\leq 0.14 \mathrm{n} \epsilon \cdot \mathrm{Hz}^{-0.5}$ or to an instantaneous strain noise standard deviation of $\leq 30 \mathrm{n} \epsilon$ at $98 \mathrm{kHz}$ data rate.

\section{Speed-of-Sound Measurement}

The potential of the proposed FSI approach for high-speed strain measurements is demonstrated through the measurement of the speed-of-sound in a stainless steel rod, as illustrated in the setup of Fig. 2. Here, following excitation by a hammer stroke, the delays in the transient response of the different segments as the impact event propagates through the rod are recorded and can be used to extract the speed-of-sound. In Fig. 10 the measured phase signals for the six segments S1 to S6 over a period of $\pm 1 \mathrm{~ms}$ around the time of the hammer impact are shown, while equivalent strain units calculated [18] for the sensor gauge length of $12.5 \mathrm{~cm}$ are additionally shown on the secondary $y$-axis. The inset in Fig. 10 then enlarges the period of the initial signal rise after the hammer impact, where propagation delays of $\approx 25 \mu \mathrm{s}$ 


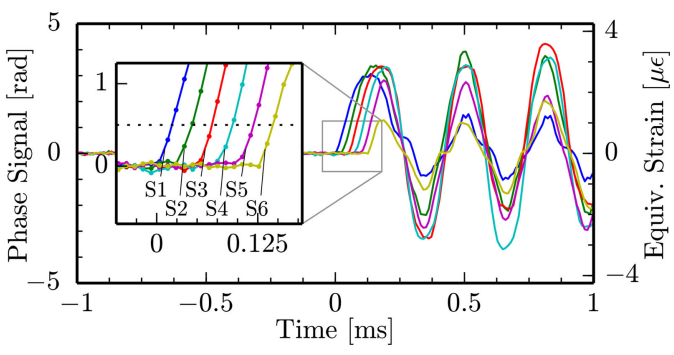

Fig. 10. Speed-of-sound measurement for the stainless steel rod with the phase signals for the six segments S1 to S6 shown at the time of the hammer impact and with the secondary $y$-axis in equivalent strain units for a segment length $l_{s}$ equal to $12.5 \mathrm{~cm}$. The inset enlarges the initial rise period and marks the threshold level at $0.5 \mathrm{rad}$, where the delay times were determined.

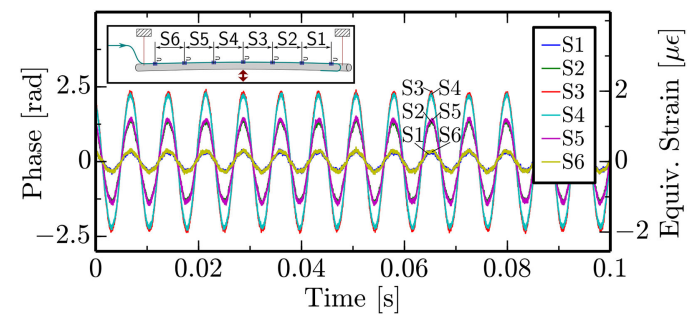

Fig. 11. Measurement over $0.1 \mathrm{~s}$ of the segment phase signals S1 to S6 after all transient events have subsided, with only the fundamental transverse mode at $137 \mathrm{~Hz}$, also illustrated in the inset, remaining. Here, the secondary $y$-axis also shows equivalent strain units for $l_{s}$ equal to $12.5 \mathrm{~cm}$.

between the segment phase signals are visible. Here, data between measurement points was linearly interpolated to allow the determination of the propagation delays at the chosen threshold level of $0.5 \mathrm{rad}$, with this threshold level also marked in the inset in Fig. 10. By evaluating the propagation delay between adjacent segments over 10 repeats of this experiment, where only the central segments S2 to S5 were used to exclude edge effects, the speed-of-sound was determined as $4.96 \pm 0.34 \mathrm{~km} \cdot \mathrm{s}^{-1}$. This is well within the error limits of the calculated theoretical value [36] of $4.91 \mathrm{~km} \cdot \mathrm{s}^{-1}$ for a longitudinal acoustic wave in a type 316 stainless steel thin rod.

Finally, Fig. 11 shows a measurement over $0.1 \mathrm{~s}$ of the six segment phase signals, recorded several seconds after the hammer impact, where all transient excitations have subsided and only the fundamental transverse vibration mode at $137 \mathrm{~Hz}$, also illustrated in the inset in Fig. 11, remains. Here, the secondary $y$-axis indicates the equivalent strain units [18] for a segment length $l_{s}=12.5 \mathrm{~cm}$. It can be seen in Fig. 11 that the signals for the outer segments S1 and S6, the signals for S2 and S5 and the signals for the inner segments S3 and S4 overlap, with the amplitude being highest for the inner segments and lowest for the outer segments. This measurement therefore agrees well with the expected behaviour for the fundamental transverse mode, with the vibration amplitudes highest in the centre and lowest at the edges, and where the mode is expected to be symmetrical about the centre of the rod. Thus, this measurement qualitatively confirms that the strain transfer onto the sensing fiber is well established, or at least comparable for all segments, and that physically plausible strain signals are detected by the FSI measurement.

\section{DISCUSSION}

In this approach the required signal processing hardware bandwidth is approximately numerically equal to the product of the modulation frequency $f_{m}$ and the phase carrier amplitude of the reflector with the largest OPD, where the resolvable quadrature bandwidth per reflector is $<0.5 f_{m}$. For the example configuration presented in this study, the reflector with the largest phase carrier amplitude can be seen in Fig. 4 to be R7 at an amplitude of $420 \mathrm{rad}$, thus requiring signal processing hardware that can resolve an interferometric fringe rate of $420 \cdot 98 \mathrm{kHz} \approx 41 \mathrm{MHz}$. Using this multiplicative relationship it can easily be seen that more sensors could be multiplexed at lower quadrature bandwidths or fewer sensors at higher bandwidths and that all figures could be improved by faster signal processing hardware. Note, however, that the required signal processing hardware bandwidth is independent of the segment length $l_{s}$ used, because, if the segment length in an FSI array is increased, a lower optical frequency excursion amplitude $f_{\text {opt }}$ can be used, resulting in the same values for the phase carrier amplitudes. In general, because the spatial resolution in the SFM technique has no fundamental limits other than $f_{\text {opt }}$, replacement of the diode laser used by a widely tunable laser would theoretically allow the interrogation of much shorter fiber segments. Also, because the FBG-based in-fiber reflectors can potentially be very short, down to below $20 \mu \mathrm{m}$ [20], there is plenty of potential to interrogate very short segment lengths in an FSI configuration. A further point to note is that in FSI, a maximum strain change rate exists beyond which the interferometric fringe count would be lost, and that this strain change rate is shared between multiple segments. With a resolvable quadrature bandwidth of $43 \mathrm{kHz}$, this results [18] in a maximum permissible strain change rate of $3.8 \cdot 10^{4} \mu \epsilon \cdot \mathrm{s}^{-1}$ for each of the six $12.5 \mathrm{~cm}$ long segments, if the strain changes are shared equally between segments. This could be improved by increasing the modulation frequency $f_{m}$.

In this paper, highly linear phase measurement with cyclic errors $\leq 7 \mathrm{mrad}$ have been demonstrated and maximum noise levels in segment signals, over the entire $43 \mathrm{kHz}$ quadrature bandwidth, did not exceed $0.17 \mathrm{mrad} \cdot \mathrm{Hz}^{-0.5}$, leading to strain noise levels of $\leq 0.14 \mathrm{n} \epsilon \cdot \mathrm{Hz}^{-0.5}$, which were found to be limited by laser phase noise. These dynamic strain resolutions are not at the performance levels of high-end applications, such as hydrophones [7], but do compare well with other commonly-used strain sensing techniques, such as FBG-based strain sensing [32], and could be improved by employing lasers with lower frequency noise levels [37].

A further point not yet addressed is the polarisation sensitivity inherent in any long-gauge length interferometric technique, potentially leading to polarisation-induced signal fading [7]. If this poses a problem, the proposed setup would allow the cost-effective use of polarisation-maintaining fiber. This is possible because this configuration avoids the use of expensive polarisation-maintaining directional couplers, while polarisation-maintaining circulators are comparable in cost to regular circulators and typical telecoms diode lasers often have polarisation-maintaining leads. 


\section{CONCLUSION}

A novel range-resolved interferometric signal processing technique has been applied to the multiplexing of long-gauge fiber optic length strain sensors, constructed using FBG-based in-fiber partial reflectors to define the fiber segments. Using a very simple optical setup with an equipment cost of below $\$ 5 \mathrm{k}$, highly linear (cyclic errors $\leq 7 \mathrm{mrad}$ ) interferometric phase measurements of six fiber segments of length $12.5 \mathrm{~cm}$, formed between seven FBGs acting as in-fiber partial reflectors, were demonstrated. Laser phase noise limited operation with noise levels in the segment signals $\leq 0.17 \mathrm{mrad} \cdot \mathrm{Hz}^{-0.5}$, equal to strain noise levels $\leq 0.14 \mathrm{n} \epsilon \cdot \mathrm{Hz}^{-0.5}$, was achieved at a data rate of $98 \mathrm{kHz}$ and a quadrature bandwidth of $43 \mathrm{kHz}$. Measurements could be taken for reflectivities as low as $1 \cdot 10^{-6}$ and it was shown that the approach is tolerant to variations in the reflector properties.

\section{ACKNOWLEDGMENT}

The authors acknowledge the support of the Engineering and Physical Sciences Research Council (EPSRC) U.K., under grants EP/H02252X/1 and EP/G033900/1. For inquiries relating to access to the research data or other materials referred to in this article, please contact Cranfield University Library and Information Services: researchdata@cranfield.ac.uk.

\section{REFERENCES}

[1] C. D. Butter and G. B. Hocker, "Fiber optics strain gauge," Appl. Opt., vol. 17, no. 18, pp. 2867-2869, 1978.

[2] A. D. Kersey, M. A. Davis, H. J. Patrick, M. LeBlanc, K. P. Koo, C. G. Askins, M. A. Putnam, and E. J. Friebele, "Fiber grating sensors," J. Lightw. Technol., vol. 15, no. 8, pp. 1442-1463, Aug. 1997.

[3] B. Glisic, "Influence of the gauge length on the accuracy of long-gauge sensors employed in monitoring of prismatic beams," Meas. Sci. Technol., vol. 22 , no. 3, p. 35206, 2011.

[4] J. P. Dakin, C. A. Wade, and M. Henning, "Novel optical fibre hydrophone array using a single laser source and detector," Electron. Lett., vol. 20, no. 1 , pp. 53-54, 1984.

[5] I. Sakai, R. C. Youngquist, and G. Parry, "Multiplexing of optical fiber sensors using a frequency-modulated source and gated output," J. Lightw. Technol., vol. 5, no. 7, pp. 932-940, Jul. 1987.

[6] G. A. Cranch and P. J. Nash, "Large-scale multiplexing of interferometric fiber-optic sensors using TDM and DWDM,' J. Lightw. Technol., vol. 19, no. 5, pp. 687-699, May 2001.

[7] C. K. Kirkendall and A. D. Dandridge, "Overview of high performance fibre-optic sensing," J. Phys. D: Appl. Phys., vol. 37, no. 18, pp. 197-216, 2004.

[8] J. A. Garcia-Souto, and H. Lamela-Rivera, "High resolution $(<1 \mathrm{~nm})$ interferometric fiber-optic sensor of vibrations in high-power transformers." Opt. Exp., vol. 14, no. 21, pp. 9679-9686, 2006.

[9] D. Vilchis-Rodriguez, S. Djurovic, P. Kung, M. I. Comanici, and A. C. Smith, "Investigation of induction generator wide band vibration monitoring using fibre Bragg grating accelerometers," in Proc. IEEE Int. Conf. Electr. Mach., 2014, pp. 1772-1778.

[10] R. Di Sante, "Fibre optic sensors for structural health monitoring of aircraft composite structures: Recent advances and applications," Sensors, vol. 15, no. 8, pp. 18666-18713, 2015.

[11] J. M. Corres, J. Bravo, F. J. Arregui, and I. R. Matias, "Unbalance and harmonics detection in induction motors using an optical fiber sensor," IEEE Sensors J., vol. 6, no. 3, pp. 605-612, Jun. 2006.

[12] W. K. Chan, G. Flanagan, and J. Schwartz, "Spatial and temporal resolution requirements for quench detection in (RE)Ba2Cu3Ox magnets using Rayleigh-scattering-based fiber optic distributed sensing," Supercond. Sci. Technol., vol. 26, no. 10, p. 105015, 2013.

[13] C. Y. Park, B.-W. Jang, J. H. Kim, C.-G. Kim, and S.-M. Jun, "Bird strike event monitoring in a composite UAV wing using high speed optical fiber sensing system," Composites Sci. Technol., vol. 72, no. 4, pp. 498-505, 2012.

[14] T. Vella, S. Chadderdon, R. Selfridge, S. Schultz, S. Webb, C. Park, K. Peters, and M. Zikry, "Full-spectrum interrogation of fiber Bragg gratings at $100 \mathrm{kHz}$ for detection of impact loading," Meas. Sci. Technol., vol. 21, no. 9, p. 094009, 2010.

[15] H. Lamela, D. Gallego, R. Gutierrez, and A. Oraevsky, "Interferometric fiber optic sensors for biomedical applications of optoacoustic imaging." J. Biophoton., vol. 4, no. 3, pp. 184-192, 2011.

[16] H. Gabai, I. Steinberg, and A. Eyal, "Multiplexing of fiber-optic ultrasound sensors via swept frequency interferometry," Opt. Exp., vol. 23, no. 15, pp. 18915-18924, 2015.

[17] T. Kissinger, T. O. H. Charrett, and R. P. Tatam, "Range-resolved interferometric signal processing using sinusoidal optical frequency modulation." Opt. Exp., vol. 23, no. 7, pp. 9415-9431, 2015.

[18] T. Kissinger, T. O. H. Charrett, and R. P. Tatam, "Fibre segment interferometry using code-division multiplexed optical signal processing for strain sensing applications," Meas. Sci. Technol., vol. 24, p. 94011, 2013.

[19] J. Huang, X. Lan, M. Luo, and H. Xiao, "Spatially continuous distributed fiber optic sensing using optical carrier based microwave interferometry." Opt. Exp., vol. 22, no. 15, pp. 18757-18769, 2014.

[20] Z. Wang, F. Shen, L. Song, X. Wang, and A. Wang, "Multiplexed fiber Fabry-Perot interferometer sensors based on ultrashort Bragg gratings," IEEE Photon. Technol. Lett., vol. 19, no. 8, pp. 622-624, Apr. 2007.

[21] Z. Chen, L. Yuan, G. Hefferman, and T. Wei, "Ultraweak intrinsic FabryPerot cavity array for distributed sensing." Opt. Lett., vol. 40, no. 3, pp. $320-323,2015$.

[22] M. E. Froggatt and J. Moore, "Distributed measurement of static strain in an optical fiber with multiple Bragg gratings at nominally equal wavelengths," Appl. Opt., vol. 37, no. 10, pp. 1741-1746, 1998.

[23] M. P. Minneman, E. Hoover, P. Boschert, J. Ensher, M. Crawford, D. Derrickson, and A. D. Kersey, "Very high sensor-density multiplexing using a wavelength-to-time domain reflectometry approach based on a rapidly swept akinetic laser," Proc. SPIE, vol. 9634, 2015.

[24] S. V. Shatalin, V. N. Treschikov, and A. J. Rogers, "Interferometric optical time-domain reflectometry for distributed optical-fiber sensing," Appl. Opt., vol. 37, no. 24, pp. 5600-5604, 1998.

[25] M. E. Froggatt and J. Moore, "High-spatial-resolution distributed strain measurement in optical fiber with Rayleigh scatter," Appl. Opt., vol. 37, no. 10 , pp. $1735-1740,1998$

[26] X. Bao and L. Chen, "Recent progress in Brillouin scattering based fiber sensors," Sensors, vol. 11, no. 4, pp. 4152-4187, 2011.

[27] T. Kissinger, R. Correia, T. O. H. Charrett, S. W. James, and R. P. Tatam, "Range-resolved signal processing for fibre segment interferometry applied to dynamic long-gauge length strain sensing," Proc. SPIE, vol. 9634, 2015.

[28] K. Itoh, "Analysis of the phase unwrapping algorithm," Appl. Opt., vol. 21, no. 14, p. 2470, 1982.

[29] C. Okawara and K. Saijyou, "Fiber optic interferometric hydrophone using fiber Bragg grating with time division multiplexing," Acoust. Sci. Technol., vol. 28, no. 1, pp. 39-42, 2007.

[30] C. E. Lee, R. A. Atkins, and H. F. Taylor, "Reflectively tapped optical fibre transversal filters," Electron. Lett., vol. 23, no. 11, pp. 596-598, 1987.

[31] E. Cibula and D. Donlagic, "Low-loss semi-reflective in-fiber mirrors," Opt. Exp., vol. 18, no. 11, pp. 12017-12026, 2010.

[32] B. Lee, "Review of the present status of optical fiber sensors," Opt. Fiber Technol., vol. 9, no. 2, pp. 57-79, 2003.

[33] P. L. M. Heydemann, "Determination and correction of quadrature fringe measurement errors in interferometers," Appl. Opt., vol. 20, no. 19, pp. 3382-3384, 1981.

[34] T. Kissinger. (2015). Range-resolved optical interferometric signal processing. Ph.D. dissertation, Cranfield Univ., Cranfield, U.K. [Online]. Available: http://dspace.lib.cranfield.ac.uk/handle/1826/9598

[35] C. K. Kirkendall, A. D. Kersey, A. D. Dandridge, M. J. Marrone, and A. R. Davis, "Sensitivity limitations due to aliased high frequency phase noise in high channel-count TDM interferometric arrays," in Proc. Int. Conf. Opt. Fibre Sensors, 1996.

[36] K. F. Graff, Wave Motion in Elastic Solids. New York, NY, USA: Dover, 2003.

[37] R. E. Bartolo, A. B. Tveten, and C. K. Kirkendall, "The quest for inexpensive, compact, low phase noise laser sources for fiber optic sensing applications," Proc. SPIE, vol. 7503, 2009.

Authors' biographies not available at the time of publication. 
2016-02-18

Fibre segment interferometry for dynamic strain measurements

Kissinger, Thomas

Institute of Electrical and Electronics Engineers

Kissinger T, Correia R, Charrett T, et al. (2016) Fibre segment interferometry for dynamic strain measurements. Journal of Lightwave Technology, Volume 34, Issue 19, October 2016, pp. 4620-4626 http://dx.doi.org/10.1109/JLT.2016.2530940

Downloaded from Cranfield Library Services E-Repository 